\title{
Steinfurth, Eva
}

\section{Die Förderung des ethischen Ernährungsbewusstseins}

Haushalt in Bildung \& Forschung 6 (2017) 1, S. 79-94

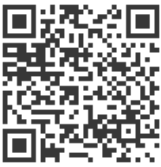

Quellenangabe/ Reference:

Steinfurth, Eva: Die Förderung des ethischen Ernährungsbewusstseins - In: Haushalt in Bildung \& Forschung 6 (2017) 1, S. 79-94 - URN: urn:nbn:de:0111-pedocs-191338 - DOI: 10.25656/01:19133

https://nbn-resolving.org/urn:nbn:de:0111-pedocs-191338

https://doi.org/10.25656/01:19133

in Kooperation mit / in cooperation with:

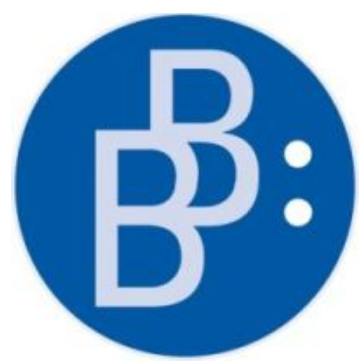

https://www.budrich.de

\section{Nutzungsbedingungen}

\section{Terms of use}

Gewährt wird ein nicht exklusives, nicht übertragbares, persönliches und beschränktes Recht auf Nutzung dieses Dokuments. Dieses Dokument ist ausschließlich für den persönlichen, nicht-kommerziellen Gebrauch bestimmt. Die Nutzung stellt keine Übertragung des Eigentumsrechts an diesem Dokument dar und gilt vorbehaltlich der folgenden Einschränkungen: Auf sämtlichen Kopien dieses Dokuments müssen alle Urheberrechtshinweise und sonstigen Hinweise auf gesetzlichen Schutz beibehalten werden. Sie dürfen dieses Dokument nicht in irgendeiner Weise abändern, noch dürfen Sie dieses Dokument für öffentliche oder kommerzielle Zwecke vervielfältigen, öffentlich ausstellen, aufführen, vertreiben oder anderweitig nutzen.

Mit der Verwendung dieses Dokuments erkennen Sie die Nutzungsbedingungen an.

We grant a non-exclusive, non-transferable, individual and limited right to using this document.

This document is solely intended for your personal, non-commercial use. Use of this document does not include any transfer of property rights and it is conditional to the following limitations: All of the copies of this documents must retain all copyright information and other information regarding legal protection. You are not allowed to alter this document in any way, to copy it for public or commercial purposes, to exhibit the document in public, to perform, distribute or otherwise use the document in public.

By using this particular document, you accept the above-stated conditions of use.

\section{Kontakt / Contact:}

\section{peDOCS}

DIPF | Leibniz-Institut für Bildungsforschung und Bildungsinformation Informationszentrum (IZ) Bildung

E-Mail:pedocs@dipf.de

Internet: www.pedocs.de

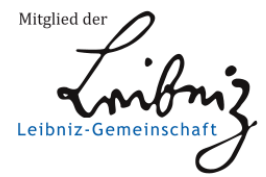


6. Jahrgang Heft 1

2017

峁

仓

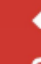

()

음

닌

亲

c

ชิ

은

\)

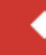

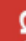

8

垔

ह

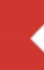

$+$

$\underline{\xi}$

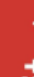

$\pm$

$\frac{9}{7}$

气

$\mathbb{1}$

চ

ISSN 2193-8806

\section{Haushalt in \\ Forschung}

\section{Qualifizierungsarbeiten Konsum - Ernährung - Gesundheit}
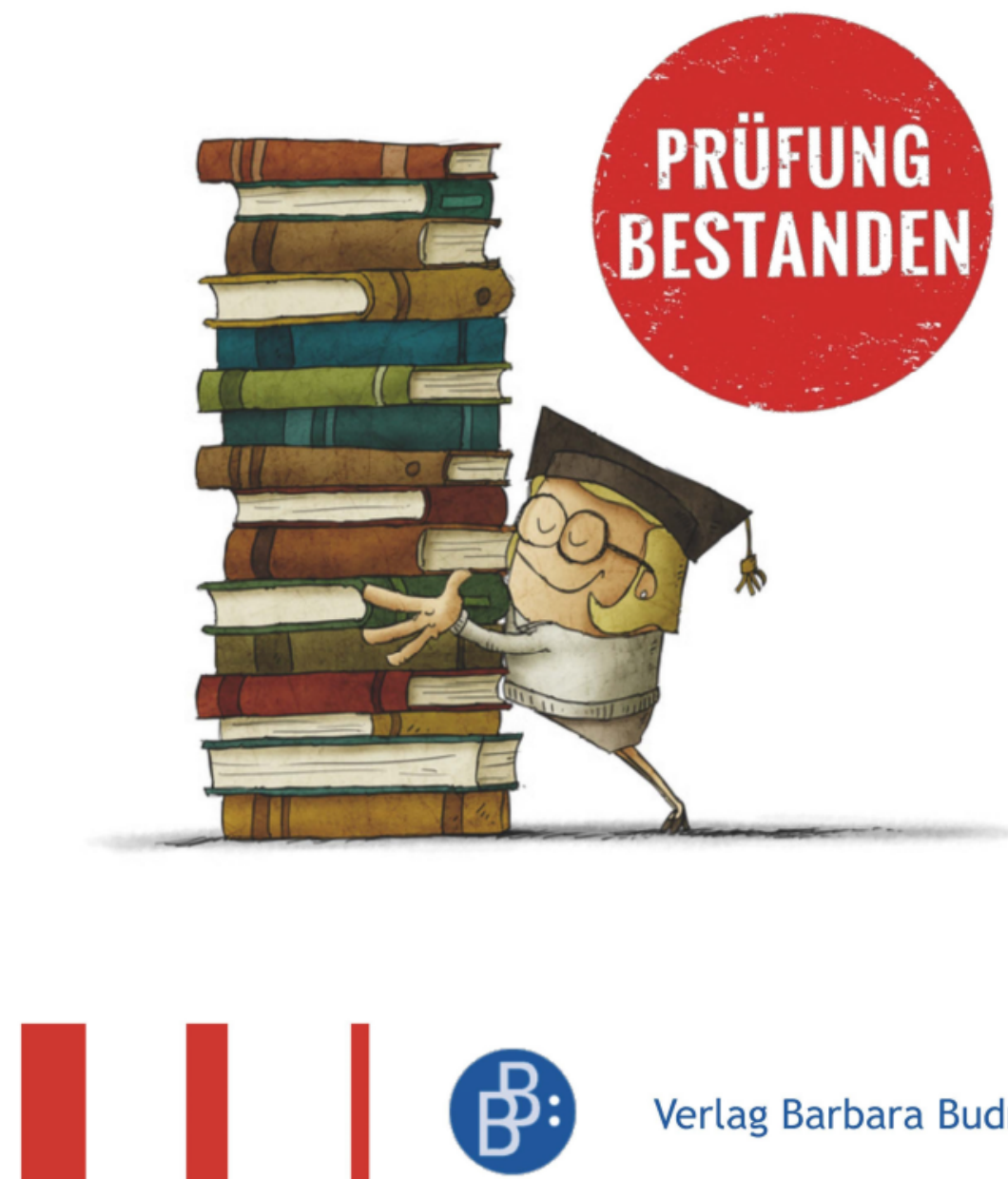

Verlag Barbara Budrich 
Inhaltsverzeichnis |

Werner Brandl

Editorial...... 2

Günther Koch

Wissenschaftliche Texte erschließen mit der Lesestrategie SQ3R 3

Martin Kornmeier

Kompetent wissenschaftlich schreiben

nach dem Gugelhupf-Prinzip.

Michael Booch

Zwischen Therapie und Beratung - Zur Rolle der Lehrkraft bei

der Präventions- und Interventionsarbeit von Essstörungen in der Schule.

Michaela Wölfer

Das Metabolische Syndrom im Jugendalter - Mögliche Ursachen

und Präventionsstrategien auf nationaler und internationaler Ebene

Julia Ernst

Aktuelle Herausforderungen bei der Schulverpflegung

an Gemeinschaftsschulen

Barbara Rieger

Essgenuss im Jugendalter.

Eva Steinfurth

Die Förderung des ethischen Ernährungsbewusstseins.

Andrea Balz \& Dina Limbach

Lernaufgaben und Unterrichtsmaterialien

zum neuen schweizerischen Lehrplan 21.

Birgit Happel

„Ich habe immer über meine Verhältnisse gelebt“ - Ein biografieanalytischer

Blick auf den Umgang mit Geld.

Tagungsankündigung

GFD Tagung 2017 Fachdidaktische Forschung zur Lehrerbildung 
Ethisches Ernährungsbewusstsein |

\section{Eva Steinfurth}

\section{Die Förderung des ethischen Ernährungsbewusstseins}

Wie kann man das ethische Ernährungsbewusstsein von Lernenden in der Zweijährigen Berufsfachschule Profil Ernährung und Hauswirtschaft fördern? Dieser Frage geht die Dokumentation einer Unterrichtseinheit nach, die im Rahmen des Referendariats an beruflichen Schulen, Baden-Württemberg, geschrieben wurde. Dabei wird sich zunächst dem Begriff des ethischen Ernährungsbewusstseins aus wissenschaftlicher Sicht angenähert. Anschließend wird mit Hilfe didaktischer und methodischer Überlegungen eine Unterrichtsreihe für die Zweijährige Berufsfachschule konzipiert, im schulischen Alltag umgesetzt und evaluiert.

Schlüsselwörter: Ernährungsbewusstsein, Ethik, Berufliche Schulen

\section{Das ethische Ernährungsbewusstsein}

Die Unterrichtseinheit hat das Ziel, das ethische Ernährungsbewusstsein der Lernenden zu fördern. Dazu wird zunächst der Zusammenhang zwischen Ernährung und Ethik aufgezeigt, um das Ziel zu rechtfertigen. Hier wird auf die Ernährungsethik zurückgegriffen, um Kriterien für das ethische Ernährungsbewusstsein abzuleiten. Weitere Kriterien lassen sich aus den Begriffen Ernährung und Bewusstsein ableiten, die ebenfalls zu diskutieren sind. Es stellt sich weiterhin die Frage, ob man das ethische Ernährungsbewusstsein als Teil der Ernährungskompetenz ansehen könnte. Sollte dies der Fall sein, könnten daraus weitere Kriterien für das ethische Ernährungsbewusstsein entstehen.

\subsection{Zusammenhang zwischen Ernährung und Ethik}

Der Zusammenhang zwischen Ernährung und Ethik wurde zum ersten Mal im Jahre 1996 von Ben Mepham in seinem Buch „Food Ethics“ diskutiert. Nach Erscheinen des Buches verbreitete sich der Terminus Ernährungsethik auch in den deutschsprachigen Ländern. Heute beschäftigen sich z. B. Studierende an der Universität Hohenheim in dem Modul "Ethics of Food and Nutrition Security“, mit vielschichtigen Fragestellungen rund um die Ernährungssicherung und werden „ebenso eingeführt in gesellschaftspolitisch relevante Themen des agrarkulturellen Wandels und der Multifunktionalität der agrarischen Systeme" (Gottwald \& Boergen, 2013, S. 13).

Um die Disziplin zu rechtfertigen musste zunächst festgestellt werden, dass Lebensmittel und somit unsere Ernährung nicht (mehr) wertfrei sind. Sie sind laut Gottwald 


\section{| Ethisches Ernährungsbewusstsein}

und Boergen ein moralisch aufgeladenes Gut. Im Vordergrund des Konsums steht nicht mehr der eigene Geschmack und die eigenen Vorlieben, sondern viel mehr die Frage, ob der eigene Konsum moralisch richtig oder falsch ist (vgl. ebd., S. 12). In jeder Kaufentscheidung schwingt unterschwellig der Wert des Lebensmittels mit bzw. was das Lebensmittel für jeden Einzelnen von uns an Wert besitzt. Und scheinbar ist das Lebensmittel den Deutschen nicht sehr viel wert. Dies lässt sich zum einen daran festmachen, dass die Bereitschaft nicht vorhanden ist, angesichts der in den letzten 50 Jahren höheren Einkommen mehr Geld für Lebensmittel auszugeben und zum anderen an den vielen Tonnen an Lebensmittelabfällen, die jährlich in Deutschland anfallen (vgl. Eberle, 2012, S. 129). So werden laut einer Studie der Universität Stuttgart, im Auftrag des Bundesministeriums für Ernährung und Landwirtschaft, 6,7 Tonnen Lebensmittel pro Jahr in der Bundesrepublik Deutschland weggeworfen (vgl. Kranert, 2012). Ein weiterer Aspekt, der hier zu nennen ist, ist die Verwendung der „Filetstücke“ und die Abneigung gegen die vollständige Verwertung der Lebensmittel (vgl. Eberle, 2012, S. 129).

Auf der anderen Seite boomt der nachhaltige Konsum, regionale Lebensmittel und Lebensmittel aus ökologischem Landbau werden scheinbar immer beliebter. Dies kann man zum Beispiel am Warenangebot verschiedener Lebensmitteldiscounter erkennen, die ihr Angebot um diese Produktgruppe in den letzten Jahren erweitert haben. Man könnte annehmen, dass sich der Wert des Lebensmittels bzw. die Einstellung einiger Menschen zum Lebensmittel und somit zu ihrer Ernährung verändert hat bzw. sich verändert.

Dies scheint auf den ersten Blick widersprüchlich, soll hier aber zur Veranschaulichung der verschiedenen Arten von Konsumenten in einer Gesellschaft dienen und aufzeigen, dass die Folgen des Konsumstils unterschiedlich sein können. Die Frage nach den Folgen des Konsumstils wird, unter anderem, in der Ernährungsethik untersucht.

Die Ernährungsethik beschäftigt sich [...] mit den Folgen der Land- und Lebensmittelwirtschaft, mit den Konsumstilen und gesellschaftspolitischen Werthaltungen. Sie beleuchtet die ökologischen, sozialen und ökonomischen Auswirkungen der Produktion, der Verarbeitung und des Konsums von Nahrung. Sie zwingt den Einzelnen zum Nachdenken über die eigenen Ernährungsgewohnheiten. (Gottwald \& Boergen, 2013, S. 14)

Die Ernährungsethik arbeitet prinzipiengeleitet. So sind die Prinzipien Vorsorge, Verantwortung, Gerechtigkeit und Vielfalt grundlegend für ein ethisches Verhalten in Lebensstilfragen und insbesondere im Bereich der Lebensmittel (vgl. ebd., S. 16).

Das Prinzip der Vorsorge plädiert darauf, Schäden zu vermeiden bevor sie entstehen. Natürlich soll dieses Prinzip den Fortschritt und die Weiterentwicklung nicht verhindern, aber die möglichen Risiken sollten Beachtung finden und berücksichtigt werden. 


\section{Ethisches Ernährungsbewusstsein |}

Das Prinzip der Verantwortung zielt zunächst auf die persönliche Verantwortung jedes Einzelnen ab und weiterhin auf die Verantwortung der Menschheit gegenüber den ökologischen Ressourcen. Es wird weiter ausgeführt, dass Verantwortung als Handlungsmaxime für ethischen Konsum durch den strategischen Konsum erreicht werden kann. Dazu muss der Verbraucher bzw. Konsument allerdings vier Stufen durchlaufen. Zunächst muss er informiert sein, dann muss er ein Bewusstsein darüber erlangen, was diese Informationen für ihn bedeuten. Anschließend gilt es, die Informationen abzuwägen. Und schließlich sollte der Konsument sich für den Kauf eines Produkts mit sozialen und ökologischen Standards entscheiden (vgl. ebd., S. 16).

Prinzip Nummer drei ist das Gerechtigkeitsprinzip. Bei Fragen rund um die Ernährung ist nicht nur die gerechte Verteilung unter den jetzt lebenden Menschen zu diskutieren, sondern auch die zwischen den jetzt lebenden Menschen und den zukünftigen Generationen.

Das letzte Prinzip bezieht sich auf die Vielfältigkeit. So trägt der Mensch dazu bei, dass nahezu jeden Tag Arten aussterben oder durch intensive Züchtung die Vielfalt der Pflanzen verringert wird. Das Gegenteil wäre aber wünschenswert: Die Erhaltung der vielfältigen Arten mit ihren jeweils eigenen Vor- und Nachteilen.

Einen kurzen Abriss über die Ernährungsethik im Wandel der Zeit findet man bei Hirschfelder und Wittmann (2015). Die interessanten Fragen einer Ernährungsethik bzw. eines ethischen Ernährungsbewusstseins kommen erst um 1990 auf. Grund dafür ist, dass der individuelle Lebensstil wichtiger wird und mit ihm die Frage nach der richtigen Ernährung. Dies führt zu der Frage nach dem ethischen Konsum im Lebensmittelbereich (vgl. ebd., S. 5).

Ausgehend von diesen Überlegungen heißt ethisch zu handeln sich seiner Verantwortung gegenüber anderen Lebewesen, aber auch der Natur, bewusst zu sein. Bezogen auf die Ernährung bedeutet das, sich seiner Verantwortung gegenüber

- der eigenen Gesundheit bewusst zu sein; die Gesundheit als Wert zu begreifen, die durch die Ernährung negativ beeinflusst werden könnte,

- den Menschen bewusst zu sein, die das Lebensmittel produzieren, d. h. sich der Arbeitsbedingungen, der Löhne, der Weiterbildungsmaßnahmen bewusst zu sein, allgemein der Behandlung der Arbeiter am Arbeitsplatz,

- den Menschen bewusst zu sein, die aufgrund von Hunger keine Perspektive haben,

- dem Lebensmittel an sich und dessen Behandlung bewusst zu sein: unser Umgang damit während der Produktion, während des Konsums und danach,

- der Umwelt bewusst zu sein; die Umwelt als Wert zu begreifen, den wir mit der Art und Weise der Lebensmittelproduktion zerstören. 


\section{| Ethisches Ernährungsbewusstsein}

So kann man von einer Dreieckbeziehung des Konsumenten, der Ernährung und der Ethik sprechen, die in Abbildung 1 ersichtlich wird.

\section{Konsumierende}

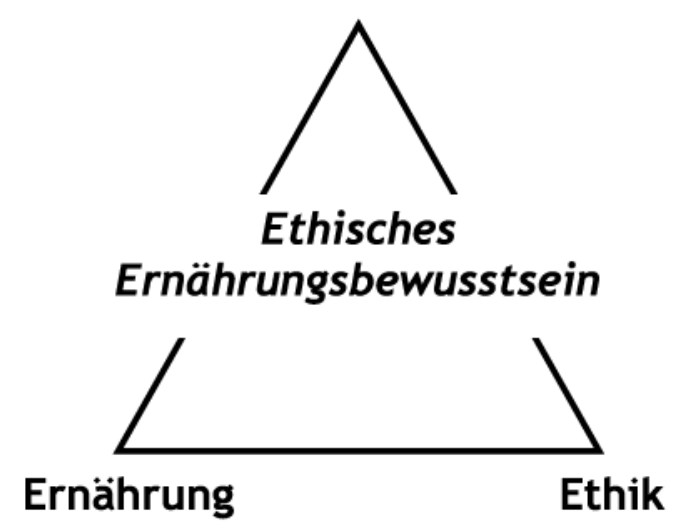

Abb. 1: Ethisches Ernährungsbewusstseinsdreieck (Quelle: eigene Darstellung)

Der Konsumierende, die Ernährung und die Ethik stehen jeweils an einer Ecke des Dreiecks. Der Konsumierende kann sich ernähren, ohne die Positionen der Ethik zu beachten, ebenso kann sich der Konsumierende mit Ethik beschäftigen, ohne dass er bzw. sie daraus Konsequenzen für die Ernährung zieht. Das Bewusstmachen der Beziehungen zwischen Konsumierenden, Ernährung und Ethik ist das Ziel, das mit der geplanten Unterrichtseinheit verfolgt werden soll.

\subsection{Ernährung und Bewusstsein}

Die Ernährung bezieht sich in erster Linie auf „das Ernähren, das Ernährtwerden, die Nahrungszufuhr und auf das Nahrung[smittel]". Somit ist die Ernährung etwas individuelles, jedem Menschen eigenes. Er muss sich ernähren bzw. ernährt werden, Energie zu sich führen, damit zunächst die Grundfunktionen wie Atmung, Herzschlag und Sauerstoffversorgung sichergestellt sind. Weiterhin, damit der Körper leistungsfähig ist. Diese Energie bekommt er aus den jeweiligen Lebensmitteln, die durch physikalische und chemische Prozesse verdaut werden. Schließlich können die energieliefernden Nährstoffe resorbiert werden. Somit wird das Nahrungsmittel zu einem Teil des Menschen, er verleibt es sich sozusagen ein. So dienen z. B. Proteine (Eiweiße) als Baustoffe für den menschlichen Körper, die je ähnlicher sie dem menschlichen Protein sind, eine höhere biologische Wertigkeit besitzen und somit ausgeschiedenes Körperprotein zu einem sehr hohen Prozentsatz ersetzen können (vgl. de Groot, 2011, S. 159 ff.). 


\section{Ethisches Ernährungsbewusstsein |}

In einer zweiten Bedeutung bezieht sich Ernährung auf die ,wirtschaftliche Versorgung“. So kann man beispielsweise von der „Ernährung der Hungernden in aller Welt sprechen“" (vgl. ebd.). Dies ist insofern relevant da die Frage nach der gerechten Verteilung der Lebensmittel mit der wirtschaftlichen Versorgung zusammenhängt. Beim Begriff des Bewusstseins wird sich auch auf die Definition des Dudens beschränkt, um eine allgemeine Sicht zu gewährleisten. Dies ist vor allem im Hinblick auf die Zielgruppe zu rechtfertigen, da diese nicht in unterschiedliche philosophische oder psychologische Strömungen eingeführt werden soll.

Bewusstsein ist laut Duden ein „Zustand, in dem man sich einer Sache bewusst ist; deutliches Wissen von etwas [hat, bzw.] Gewissheit“ aber auch die „Gesamtheit der Überzeugungen eines Menschen, die von ihm bewusst vertreten werden“.

Im Rahmen der vorliegenden Arbeit soll vom Ernährungsbewusstsein dann gesprochen werden, wenn man

- über Kenntnisse die Ernährung und die Nahrungsmittel betreffend verfügt und

- sich der Sache „Ernährung“ bzw. der Sache „Lebensmittel“ bewusst ist.

Somit sind zwei grundlegende Forderungen an die Unterrichtseinheit zu stellen:

1. Die fachwissenschaftlichen Grundlagen müssen erarbeitet werden.

2. Die Dimension des „Bewusstwerdens“ muss angestrebt werden.

Die Dimension des Bewusstwerdens kann erreicht werden, wenn die Lernenden dazu angehalten werden, sich mit verschiedenen Fragen rund um ihr eigenes Konsumverhalten zu beschäftigen. Sie informieren sich über die Produktionsbedingungen, die Arbeitsbedingungen, die Auswirkungen der Lebensmittel auf ihren Körper und auf die Umwelt und entscheiden sich dann ggf. für ein Produkt mit sozialen und ökologischen Standards. Grundlage für ein Bewusstsein in Fragen Ernährung und Lebensmittel ist die Fähigkeit Zusammenhänge herzustellen (vgl. Tuttle, 2014, S. 14).

Durch die Bewertung von Handlungen im Hinblick auf den Umgang mit der eigenen Ernährung und den Lebensmitteln befinden wir uns auf einer anderen Ebene. Die ethische Dimension wird angestrebt und konkretisiert sich im ethischen Konsum, der durch den strategischen Konsum mit seinen vier Bausteinen erreicht werden könnte (siehe oben).

Die Bewusstseinsebene kommt noch mehr zum Tragen, wenn die Lernenden ein übergreifendes Wissen bezüglich der Ernährung und Ethik haben, sich die Überzeugungen manifestieren und sie diese auch im häuslichen Bereich vertreten.

\subsection{Ethisches Ernährungsbewusstsein: Kriterien}

Das ethische Ernährungsbewusstsein setzt sich also aus mehreren Komponenten zusammen: 


\section{| Ethisches Ernährungsbewusstsein}

- Es sind fachwissenschaftliche Grundlagen zu Ernährung und Lebensmittel zu vermitteln.

- Es gilt den Lernenden als Konsument anzusprechen, der bereits eine Konsumentenrolle übernommen und eingenommen hat.

- Es ist der Zusammenhang zwischen Ernährung und Ethik darzustellen.

- Es gilt das Bewusstsein der Lernenden für die Frage nach einer ethisch korrekten Ernährung zu sensibilisieren.

Anhand dieser Kriterien kann der Lernerfolg der Unterrichtseinheit am Ende evaluiert werden. Bei einer Leistungskontrolle könnten die fachwissenschaftlichen Inhalte als auch die Zusammenhänge zwischen Ernährung und Ethik abgeprüft werden. Das Bewusstsein für eine ethisch korrekte Ernährung kann ebenfalls durch Schüleräußerungen überprüft werden. Eine Veränderung der Konsumentenrolle und ein nachhaltig verändertes Bewusstsein gegenüber der eigenen Ernährung bzw. gegenüber Nahrung kann nicht überprüft werden, da dies einen längerfristigen, auch außerschulischen, Lernprozess darstellt.

Als nächstes soll der Begriff der Ernährungskompetenz dargestellt und mögliche Verbindungen zum ethischen Ernährungsbewusstsein aufgezeigt werden.

\subsection{Ethisches Ernährungsbewusstsein und Ernährungskompetenz}

Die Bedeutung der Ernährungskompetenz lässt sich daran festmachen, dass eine erste Definition nach dem Katalyse-Institut für angewandte Umweltforschung bereits überarbeitet worden ist (vgl. Büning-Fessel, 2011, S. 6). Demnach verstand man unter Ernährungskompetenz „die Fähigkeit, theoretische und praktische Fertigkeiten im Ernährungsalltag in ein angemessenes Handeln umzusetzen, zum Beispiel im Sinne einer gesunden und nachhaltigen Ernährung" (ebd., S. 6).

Heute spricht man von der Ernährungskompetenz als Schlüsselkompetenz, die mit dem Begriff Food Literacy in Fachzeitschriften zu finden ist:

Food Literacy ist die Fähigkeit, den Ernährungsalltag selbstbestimmt, verantwortungsbewusst und genussvoll zu gestalten. „Selbstbestimmt“ meint, dass eine Person ihren Essalltag bewusst gestalten und alleine bestimmen kann. „Verantwortungsbewusst" bezieht sich einerseits auf den Umgang mit dem eigenen Körper, also darauf Verantwortung für sich und die eigene Gesundheit zu übernehmen. Es bezieht sich aber auch auf die Verantwortung für andere [...]. (ebd., S. 6)

Vergleicht man diese Definition mit den Zielen der Ernährungsethik kann man feststellen, dass es eine Übereinstimmung im Bereich der Verantwortung gibt. Wie oben bereits ausgeführt soll sich der Mensch bezogen auf die Ernährung seiner Verantwortung gegenüber der eigenen Gesundheit, der Menschen, die das Lebensmittel produzieren, den Menschen, die aufgrund von Hunger keine Perspektive haben, den Le- 


\section{Ethisches Ernährungsbewusstsein |}

bensmitteln und gegenüber der Umwelt bewusst sein. Dies lässt sich auch aus der Ernährungskompetenz ableiten.

Die Fähigkeit, den Ernährungsalltag selbstbestimmt und genussvoll zu gestalten, wird nicht explizit in der Ernährungsethik aufgegriffen, lässt sich darin aber implizit wiederfinden. Schließlich handelt man selbstbestimmt, wenn man sich über die Bedingungen der Ernährung bzw. der Lebensmittelproduktion informiert, den Lebensmittelkonsum aus ethischer Sicht bewertet und Konsequenzen daraus zieht. Auch der Genuss findet sich letztendlich darin wieder, wenn man davon ausgeht, dass sich der Genuss nicht nur im Geschmack des Lebensmittels wiederfindet, sondern auch in der Zufriedenheit des Menschen bezüglich seiner ethisch korrekten Ernährungsweise.

Es kann also gesagt werden, dass sich die Ernährungskompetenz als Schlüsselkompetenz in den dargestellten Überlegungen zur Ernährungsethik und des Ernährungsbewusstseins wiederfindet und sie sich nicht widersprechen, sondern eher überlappen. Für die Erziehungs- und Bildungsarbeit an der Schule ist das besonders hervorzuheben. Denn somit lässt sich die Ernährungskompetenz, die ausdrücklich im Vorwort des Lehrplans Berufsfachliche Kompetenz der 2BFS gewünscht ist, fächerübergreifend durch das gewählte Thema „Förderung des ethischen Ernährungsbewusstseins“ im Fach Ethik unterstützen.

\section{Didaktische Analyse}

In der didaktischen Analyse werden zunächst die anthropogenen und soziokulturellen Voraussetzungen der Lerngruppe vorgestellt. Des Weiteren soll durch den Punkt Lehrplanbezug die Unterrichtseinheit in den Lehrplan eingeordnet werden. Die didaktischen Vorüberlegungen verweisen auf das didaktische Konzept des Ethikunterrichts in der Zweijährigen Berufsfachschule (2BFS). Methodische Überlegungen runden die Analyse ab.

\subsection{Anthropogene und soziokulturelle Voraussetzungen}

Die 2BFS1 (1. Jahr) setzt sich aus 33 Lernenden zusammen. 18 (12 weiblich und 6 männlich) davon besuchen den Ethikunterricht. Die Lerngruppe ist sehr heterogen. Das beginnt beim Alter und hört beim Kenntnisstand der deutschen Sprache auf. Die Lernenden sind zwischen 15 und 20 Jahren alt. Acht davon sind in Deutschland geboren und aufgewachsen, wobei lediglich zwei keinen Migrationshintergrund vorweisen. Ein Lernender ist bereits seit acht Jahren in Deutschland, die anderen neun befinden sich erst seit kurzen in Deutschland. Alle verfügen über einen abgeschlossenen Hauptschulabschluss und erhoffen sich durch den Besuch der zweijährigen zur Fachschulreife führenden Berufsfachschule bessere Chancen auf dem Arbeitsmarkt. Es ist hinzuzufügen, dass mancher Schulabschluss in einem anderen Land erworben wurde, was die Vergleichbarkeit der Abschlüsse erschwert. Auf die Frage, warum sie 


\section{| Ethisches Ernährungsbewusstsein}

am Ethikunterricht teilnehmen antwortete die Gruppe recht homogen: weil sie weder katholisch noch evangelisch getauft worden seien.

Die Lerngruppe scheint im Großen und Ganzen gut miteinander auszukommen. Hervorzuheben ist, dass der Gruppenälteste eine Art Führungsposition übernimmt und oft den Anfang macht, bevor die anderen ebenfalls beginnen. Auch scheint eine Person aufgrund ihrer ruhigen, zurückhaltenden Art von den anderen Gruppenmitgliedern ausgeschlossen zu werden.

Die spärlichen Kenntnisse der deutschen Sprache sind ein weiteres Problem, das den Unterricht oft stocken lässt, da die Texte bzw. Wörter nicht verstanden werden oder die Lerngruppe den Arbeitsauftrag nicht versteht. Einige bringen stets ein Wörterbuch mit, um unbekannte Worte nachzuschlagen. Das braucht Zeit und wird in der Planung des Unterrichts berücksichtigt.

\subsection{Lehrplanbezug}

Die Unterrichtseinheit wird in der 2BFS1 im Rahmen des Ethikunterrichts in der Lehrplaneinheit 10 Konsum und Kaufvergnügen durchgeführt. Im Unterricht sollen die Lernenden vorwiegend mit

- den Auswirkungen des Konsums von Nahrung und

- dem Nachdenken über ihre eigenen Ernährungsgewohnheiten bzw. Kaufgewohnheiten konfrontiert werden.

Diese Auswahl wird getroffen, um die Förderung des ethischen Ernährungsbewusstseins in der Lehrplaneinheit 10 Konsum und Kaufvergnügen der 2BFS1 zu rechtfertigen und verorten zu können.

\subsection{Didaktische Vorüberlegungen}

Die Lerninhalte werden in Abhängigkeit von der Lerngruppe und dem Unterrichtsmodell des Ethikunterrichts für die zweijährige zur Fachschulreife führende Berufsfachschule gewählt. Das Unterrichtsmodell des Ethikunterrichts als Lebenshilfe stellt den Lernenden mit seinen situativen und existentiellen Lebensproblemen in den Mittelpunkt (vgl. Pfeiffer, 2009, S. 28 f.). Ziel ist die Selbstfindung des Individuums, hier mit dem Schwerpunkt der Gestaltung und Selbstbestimmung im Hinblick auf die Ernährung, als auch die Gestaltung seines Sozialverhaltens, das ebenfalls die Konsumentscheidungen hinsichtlich des Essens und Trinkens beeinflussen könnte.

Aufgrund des Unterrichtsmodells und der Tatsache, dass sich die Lernenden in einer Entwicklungsphase vom Jugendlichen zum Erwachsenen befinden, werden die Unterrichtsinhalte so gewählt, dass sie mit der Lebenswelt der Lernenden zusammenhängen und die Entwicklung hin zu einer verantwortungsvollen Person in der Gesellschaft unterstützen. 


\section{Ethisches Ernährungsbewusstsein |}

Grundlegend für die Auswahl der Inhalte ist darüber hinaus, dass die Lernenden ihre eigenen Erfahrungen bezüglich ihres Ess- bzw. Konsumverhaltens einbringen und daran ihr ethisches Ernährungsbewusstsein schulen können.

Auch bei den Prinzipien wird ein Schwerpunkt gewählt. Hier soll der Fokus auf dem Prinzip der Verantwortung liegen. Meines Erachtens kann man den Jugendlichen sehr gut in seiner Rolle als Konsumierender ansprechen und er bzw. sie kann sich in dieser Position seiner bzw. ihrer Verantwortung bewusstwerden. Die obige Darstellung des strategischen Konsums kann für die Unterrichtsplanung ebenfalls herangezogen werden, da die Lernenden erst nach einer Phase der Information ein Bewusstsein erlangen können, aufgrund dessen sie abwägen und sich für ein Produkt mit sozialen und ökologischen Standards entscheiden können.

Die Förderung des ethischen Ernährungsbewusstseins ist dann also erreicht, wenn die Lernenden sich im Klaren sind, ihnen also bewusstwird, dass sie sich erst informieren müssen, um dann eine angemessene Entscheidung hinsichtlich ihres Ernährungsstils und ihre Konsums bezogen auf die „Wertigkeit“ des Lebensmittels treffen zu können. Wenn dieser Schritt in der 2BFS1 erfolgreich ist, so kann man sagen, dass das ethische Ernährungsbewusstsein gefördert wurde.

Somit werden für die geplante Unterrichtseinheit folgende Themen ausgewählt (siehe Tabelle 1), die das ethische Ernährungsbewusstsein in der 2BFS1 fördern sollen:

Tab. 1: Themenauswahl für die Unterrichtsstunden (je 90 Minuten)

\begin{tabular}{c|l}
\hline Stunde & \multicolumn{1}{c}{ Thema } \\
\hline 1 und 2 & Zusammenhänge zwischen Ernährung und Ethik \\
\hline 3 und 4 & Werbung, Konsum, Überfluss \\
\hline 5 und 6 & Verschwendung hier, Armut da \\
\hline 7 und 8 & Was kaufe ich ein? Oder: Der ethische Konsum \\
\hline 9 & Leistungskontrolle/Evaluation \\
\hline
\end{tabular}

\subsection{Methodische Vorüberlegungen}

Der Ethikunterricht bietet viele Möglichkeiten aus dem bekannten Methodenrepertoire zu schöpfen. Da die Lerngruppe sehr lebhaft ist und klare Anweisungen braucht, wird auf Methoden zurückgegriffen, die aus der bisherigen Schullaufbahn bekannt sein müssten und die während der Unterrichtseinheit nicht erst eingeführt werden müssen. Hierzu gehören die Arbeit mit Texten, die Arbeit mit Mindmaps sowie die Gruppenarbeit. Neben Arbeitsphasen in der Gruppe oder im Plenum spielt die Einzelarbeit eine wichtige Rolle. Diese ist für die eigenständige Beschäftigung 


\section{| Ethisches Ernährungsbewusstsein}

mit den jeweiligen Fragestellungen sehr wichtig und fördert die Urteilsfähigkeit der Lernenden. An die Einzelarbeit schließt stets eine Auswertungsphase im Plenum an, in der die Ergebnisse besprochen und verglichen werden können. Dies ist wichtig, da die Lernenden stets die Gewissheit haben möchten, ob sie den Arbeitsauftrag angemessen bearbeitet haben. Auch können hier Urteile offengelegt, überdacht und gegebenenfalls neu formuliert werden.

In einigen Unterrichtsstunden wird mit einem Film gearbeitet. Das bietet sich insofern an, da im Film komplexe Sachverhalte gezeigt werden können, die anhand anderer Medien nur schwer zu veranschaulichen sind. Der Film wird zielführend für die weitere Erarbeitung im Unterricht durch entsprechende Arbeitsaufträge genutzt. Der Lehrervortrag wird nur selten eingesetzt, da die Lerngruppe sehr lebhaft ist und einem längeren Vortrag nur kurzfristig Aufmerksamkeit schenken kann. Wird der Lehrervortrag eingesetzt, so sollte er mit Bildern oder ähnlichem unterstützt werden, so dass die Lernenden während des Zuhörens durch den Vortrag geleitet werden.

\section{Konkrete Unterrichtsplanung}

Die Dokumentation umfasst vier Doppelstunden (90 Minuten) und eine Einzelstunde (45 Minuten), die zur Evaluation der Unterrichtseinheit dient. Jede Darstellung bezieht sich zunächst auf die Ziele und gibt dann einen Überblick über die einzelnen Phasen der geplanten Unterrichtsstunden. Hier soll sich auf die Darstellung der ersten Unterrichtseinheit beschränkt werden. ${ }^{1}$

\section{Erste Doppelstunde - Zusammenhänge zwischen Ernährung und Ethik}

Einstieg: Die Begriffe Ethik und Ernährung werden nacheinander von der Lehrperson an die Tafel geschrieben und die Klasse bekommt den Auftrag ihre Assoziationen zu den Begriffen wie bei einer Mindmap an die Tafel zu schreiben. Die Methode soll das freie Denken der Lernenden fördern und sie dazu motivieren, sich am Unterricht zu beteiligen. Da zunächst alles ohne Wertung gesammelt wird, gibt es kein richtig und falsch. Gleichzeitig bietet sich hier die Möglichkeit, den Erfolg der vorangegangenen Unterrichtseinheit zum Thema „Was ist Ethik?“ zu evaluieren.

Jeder Begriff wird auf eine eigene Tafelseite geschrieben und nacheinander bearbeitet. Das Vorgehen hat das Ziel, die Begriffe Ethik und Ernährung jeweils einzeln zu betrachten und das Wissen der Lernenden zu den jeweiligen Begriffen abzufragen. Somit werden die Gedanken der Lernenden offengelegt und man kann gut daran anknüpfen bzw. damit weiterarbeiten.

Problemerarbeitung: Nach der offenen Phase, in der zwei Mindmaps erarbeitet werden, wird die Klasse mit einem aktuellen Zeitungsartikel konfrontiert, der mit dem Titel: „Ethik und Essen: Jeder Biss eine gute Tat“ (Block, 2015) den Zusam- 


\section{Ethisches Ernährungsbewusstsein |}

menhang zwischen Ethik und Ernährung thematisiert. Der Artikel zeigt, dass das Thema sehr aktuell ist und passt deshalb sehr gut an den Beginn der Unterrichtseinheit. Auch merken die Lernenden, dass das Thema von einer breiten Öffentlichkeit diskutiert wird und keinesfalls abstrakt ist. Zudem könnte der Zeitungsartikel die Lernenden zum Zeitungslesen animieren. Da dieser sehr lang ist, wird der Artikel zusammen mit den Lernenden gelesen. Es wird genügend Zeit eingeplant, damit die Lernenden bei unbekannten Wörtern nachfragen können und so jeder den Text verstehen kann. Nach einem ersten gemeinsamen Lesen haben die Lernenden in Einzelarbeit Zeit, den Text noch einmal durchzulesen und die Arbeitsaufträge zu bearbeiten. Das ist wichtig, da die Klasse sehr heterogen ist und einige länger brauchen, um einen Text zu erfassen als andere. Da dies aber die Einführungsstunde des neuen Themas ist und mit dem Text im Anschluss noch gearbeitet werden soll, ist es wichtig, dass alle den Text gelesen und verstanden haben, weshalb hier viel Zeit investiert wird.

Auswertung: Im Plenum wird der erste Arbeitsauftrag besprochen und die Lerngruppe hat Zeit, ihre Ergebnisse zu vergleichen. Der Text wird über den Präsenter sichtbar gemacht und die entsprechenden Stellen werden farbig markiert bzw. unterstrichen. Der zweite Teil des Arbeitsauftrages wird ebenfalls im SchülerLehrer-Gespräch im Plenum beantwortet und an der Tafel fixiert. Der zweite Arbeitsauftrag ist auch als eine Art Puffer für die leistungsstärkeren Lernenden gedacht. Deren Ausführungen werden ggf. etwas abgeändert an die Tafel genommen und als Tafelbild von der Lerngruppe übernommen. Somit werden die Leistungsstärkeren bestärkt und die ganze Gruppe kann davon profitieren.

Reflexion: Nach der intensiven Textarbeit und der Auswertung dieser wird auf die Mindmaps vom Anfang der Stunde zurückgegriffen. Ausgehend vom Text sollen die Lernenden nochmals über beide Mindmaps nachdenken und weitere Aspekte die die Themenbereiche Ethik und Ernährung betreffen aufzeigen und ergänzen. Somit dient der Text als Erweiterung der Gedankenwelt der Lernenden neue Aspekte oder Ansichten könnten zur Sprache kommen und aufgenommen werden. Zum Schluss der Stunde werden diejenigen Aussagen an der Tafel eingerahmt und dadurch hervorgehoben, die beim Thema Ernährung und Ethik eine große Relevanz haben und die in den kommenden Stunden vertieft werden sollen.

\section{Reflexion}

Nachdem die theoretische Planung vorgesellt wurde, soll nun anhand der Reflexion diskutiert werden, welche Planungsschritte erfolgreich waren und an welchen Stellen Verbesserungen oder gar Änderungen wünschenswert sind. Ebenfalls ist zu überprüfen, ob die Lernziele der jeweiligen Stunden erreicht worden sind und ob das angestrebte Ziel, nämlich die Förderung des ethischen Ernährungsbewusstseins in der 


\section{| Ethisches Ernährungsbewusstsein}

2BFS1 erfolgreich war. Die Reflexion wird hier für die erste Doppelstunde als auch für die gesamte Unterrichtseinheit dargelegt.

\subsection{Reflexion - Erste Doppelstunde}

Die erste Doppelstunde war geprägt von der Arbeit mit den Mindmaps. Zunächst verlief diese Phase sehr zäh, da die Lernenden es nicht gewohnt sind, sich eigene Gedanken zu machen. Sie haben Angst etwas Falsches zu sagen oder gar an die Tafel zu schreiben. Mit der Zeit aber wurde die Lerngruppe mutiger und es wurden viele Gedanken zu den zwei Themenbereichen Ernährung und Ethik gesammelt. Die Lernenden waren angehalten, selbst ihre Gedanken an die Tafel zu bringen, was die Mindmap zum Teil sehr unübersichtlich machte. Eventuell wäre die Arbeit mit Metaplankarten besser gewesen, da man diese im Anschluss besser hätte strukturieren können.

Es folgte die Textarbeit. Der Text „Ethik und Essen: Jeder Biss eine gute Tat" (Block, 2015) ist einer Zeitung entnommen und relativ lange. Vom Niveau her war er passend für einen Teil der Lerngruppe. Diejenigen, die erst seit kurzem in Deutschland sind hatten Schwierigkeiten den Text zu verstehen. Das gemeinsame Lesen des Textes war sehr gut, da hier auch diejenigen mit weniger Deutschkenntnissen einen ersten Überblick bekamen. Die Beschäftigung mit dem Text in Einzelarbeit war unbedingt nötig, hat aber viel Zeit in Anspruch genommen. Das gemeinsame Besprechen des Textes im Plenum war ebenfalls gut gewählt, da hier die Ergebnisse verglichen werden konnten. Der letzte Arbeitsauftrag wurde nur von den leistungsstärkeren Lernenden bearbeitet. Es war gut, dass es einen weiteren Arbeitsauftrag gab, denn sonst wären diese lange Zeit ohne Beschäftigung gewesen.

Nachdem der Text gelesen wurde, sollten die Lernenden neue oder andere Gedanken aus dem Text, die zum Thema Ernährung oder Ethik passten, in der Mindmap ergänzen. Hier wurde beobachtet, dass sich die Lernenden sehr schwer tun, das Gelesene mit dem vorher Besprochenen zu Verknüpfen. Es dauerte einige Zeit, bis der Arbeitsauftrag verstanden wurde und die Mindmaps ergänzt werden konnten. Eventuell hätte es sich angeboten, die Aussagen aus dem Text nicht nur zu markieren, sondern herauszuschreiben und ebenfalls in einem Schaubild zu veranschaulichen. Dann wären die neuen oder weiteren Gedanken den Lernenden mehr ins Auge gefallen und sie hätten sich leichter getan, die Mindmap zu ergänzen. Insgesamt hätte man mehr mit den Aussagen des Textes arbeiten können. Er enthält viele sehr gute Aussagen, die zwar markierten worden sind, mit denen aber nicht weitergearbeitet wurde. Darauf könnte man zurückführen, dass die Mindmap vom Beginn sich nicht sehr von der Mindmap am Ende der Stunde unterscheidet. Insgesamt muss noch mehr mit den Materialien gearbeitet werden, die in die Lerngruppe gegeben werden. Die Herstellung einer Verbindung zwischen den Begriffen Ethik und Ernährung fand statt. Diese hätte aber noch viel klarer herausgearbeitet und vertieft werden können. 
Ethisches Ernährungsbewusstsein |

\subsection{Reflexion der Unterrichtseinheit}

Die Unterrichtseinheit setzte sich zusammen aus vier Doppelstunden und einer Evaluationsstunde.

Die Unterrichtsstunden sind insgesamt logisch aufeinander aufgebaut. Wo es Schwierigkeiten bei den Überlegungen und der Umsetzung gab ist die zweite Doppelstunde. Hier wurde sich noch sehr stark am Lehrplan orientiert, der Werbung und Konsum als Themen anbot. Der Übergang innerhalb der Doppelstunde zum Filmausschnitt „Leben im Überfluss“ war nicht gelungen, weil es thematisch schwierig war, an das vorherige Thema Werbung anzuknüpfen. Von der Werbung sollte aber auf den Konsum und die Konsumgesellschaft verwiesen werden. Nur wer sich unserer Konsumgesellschaft bewusst ist, kann sie auch kritisieren und versuchen alternative Wege zu gehen. Dies ist meines Erachtens nicht gut gelungen und gibt der Unterrichtseinheit einen kleinen Bruch. Die erste Doppelstunde als große Einführungsstunde in den Themenbereich Ernährung und Ethik ist mit der Wahl der Mindmaps und dem aktuellen Artikel gut gelungen. Sie gibt einen ersten Überblick und schafft ein Interesse für das Thema und eruiert die Ansichten der Lerngruppe. Ebenfalls zufrieden bin ich mit den Doppelstunden „Verschwendung hier, Armut da“ und „Was kaufe ich ein? oder Der ethische Konsum“. Beide Doppelstunden sind klar gegliedert, enthalten einen roten Faden, der sich auch durch die Unterrichtseinheit zieht. Am Schluss wird der ethische Konsum, der als verantwortungsvolles Handeln unter den vier Prinzipien der Ernährungsethik dargestellt wurde, thematisiert. Den ethischen Konsum als Schlüsselthema der ganzen Unterrichtseinheit hätte man noch viel tiefer besprechen müssen. Er hätte die zentrale Stellung in der Planung einnehmen müssen. Auf der anderen Seite müssen aber erst einmal Grundlagen geschaffen werden, durch die es möglich ist, auf den ethischen Konsum zu sprechen zu kommen. Dieser Versuch ist in der Unterrichtseinheit unternommen worden. Eine weitere Einheit zu Förderung des ethischen Ernährungsbewusstseins könnte den ethischen Konsum beispielsweise gleich in der ersten Stunde thematisieren und daran anschließend verschiedene Produkte besprechen und beurteilen.

Die Unterrichtseinheiten sind teils schülernah geplant, manche Einheit könnte jedoch noch mehr die Lernenden und Thre Bedürfnisse im Blickfeld haben. Auch diesen Aspekt könnte man bei einer nochmaligen Durchführung der Unterrichtseinheit verbessern und ausgehend von alltäglichen Konsumentscheidungen der jungen Konsumierenden verschiedenen Produkte unter die Lupe nehmen und deren Produktion bzw. Herstellungsverfahren besprechen und nach ethischen Kriterien bewerten.

\section{Fazit}

Die Ernährungsethik ist eine relativ junge Forschungsdisziplin, deren Gegenstand es ist, die Folgen der Land- und Lebensmittelwirtschaft, die Folgen unseres Konsum- 


\section{| Ethisches Ernährungsbewusstsein}

stils als auch unsere gesellschaftspolitische Werthaltung gegenüber Lebensmitteln zu untersuchen. Des Weiteren soll der Verbraucher dazu gebracht werden, sich mit seinem Lebensmittelkonsum kritisch auseinanderzusetzen. Dafür wurden vier Prinzipien aufgestellt, nach denen die Ernährungsethik arbeitet. Für den Unterricht war das Prinzip der Verantwortung richtungsweisend, das man auch in zukünftigen Unterrichtsstunden weiterverfolgen und vertiefen könnte.

Es wurde gezeigt, dass die Förderung des ethischen Ernährungsbewusstseins in der 2BFS1 im Fach Ethik wünschenswert ist, da hier die Nahrung bzw. die Lebensmittel nicht nur aus einer naturwissenschaftlichen Sichtweise heraus untersucht werden, wie es in der Berufsfachlichen Kompetenz (BFK) der Fall ist. Somit trägt das ethische Ernährungsbewusstsein zur Förderung der Ernährungskompetenz bei, die als Schlüsselkompetenz im Lehrplan der 2BFS gefordert wird.

Bei der fachwissenschaftlichen Auseinandersetzung wurde aufgezeigt, dass sich die Verantwortung bezogen auf Ernährung auf folgende Aspekte beziehen könnte:

- sich der eigenen Gesundheit bewusst zu sein; die Gesundheit als Wert zu begreifen, die durch die Ernährung negativ beeinflusst werden könnte,

- sich den Menschen bewusst zu sein, die das Lebensmittel produzieren, d. h. sich der Arbeitsbedingungen, der Löhne, der Weiterbildungsmaßnahmen bewusst zu sein, allgemein der Behandlung der Arbeiter am Arbeitsplatz,

- sich den Menschen bewusst zu sein, die aufgrund von Hunger keine Perspektive haben,

- sich dem Lebensmittel an sich und dessen Behandlung bewusst zu sein: unser Umgang damit während der Produktion, während des Konsums, und danach,

- sich der Umwelt bewusst zu sein; die Umwelt als Wert zu begreifen, den wir mit der Art und Weise der Lebensmittelproduktion zerstören.

Da man in einer Unterrichtseinheit von sechs bis acht Stunden immer eine Auswahl treffen muss sind folgende Aspekte nicht berücksichtigt worden:

- $\quad$ sich der eigenen Gesundheit bewusst zu sein; die Gesundheit als Wert zu begreifen, die durch die Ernährung negativ beeinflusst werden könnte,

- sich den Menschen bewusst zu sein, die aufgrund von Hunger keine Perspektive haben,

- sich der Umwelt bewusst zu sein; die Umwelt als Wert zu begreifen, den wir mit der Art und Weise der Lebensmittelproduktion zerstören.

Der Schwerpunkt der Unterrichtseinheit lag bei der Förderung des ethischen Ernährungsbewusstseins auf den beiden Aspekten:

- sich den Menschen bewusst zu sein, die das Lebensmittel produzieren, d. h. sich der Arbeitsbedingungen, der Löhne, der Weiterbildungsmaß- 


\section{Ethisches Ernährungsbewusstsein |}

nahmen bewusst zu sein, allgemein der Behandlung der Arbeiter am Arbeitsplatz,

- sich dem Lebensmittel an sich und dessen Behandlung bewusst zu sein: unser Umgang damit während der Produktion, während des Konsums, und danach,

Somit wurden auch die Komponenten des ethischen Ernährungsbewusstseins erreicht, die da lauten:

- Es ist der Zusammenhang zwischen Ernährung und Ethik darzustellen.

Das wurde in der ersten Stunde zur Förderung des ethischen Ernährungsbewusstseins herausgearbeitet. In der Unterrichtseinheit wurden oben genannte Aspekte vertieft.

- Es gilt den Lernenden als Konsument anzusprechen, der bereits eine Konsumentenrolle übernommen und eingenommen hat.

Dies wurde durch die Konfrontation mit den Themen Werbung und Konsum sowie Leben im Überfluss angesprochen und aufgezeigt.

- Es gilt das Bewusstsein der Lernenden für die Frage nach einer ethisch korrekten Ernährung zu sensibilisieren.

Vor allem in der letzten Stunde wurden die Lernenden mit dem ethischen Konsum, wenn auch zunächst unbewusst konfrontiert. Eine Sensibilisierung, ein Bewusstmachen hat stattgefunden. Ob es von Dauer ist, kann nicht gesagt werden.

Obwohl alle drei Komponenten im Rahmen der Unterrichtseinheit angesprochen wurden und somit als Indikator für den Erfolg der Unterrichtseinheit verbucht werden könnten, muss hier auf die Reflexion der Unterrichtsstunden verwiesen werden und die darin enthaltenen Kritikpunkte.

Fachwissenschaftliche Grundlagen zu Ernährung und Lebensmittel sind in der vorliegenden Unterrichtseinheit nicht vermittelt worden. Dies hat sich aus der Planung heraus nicht ergeben, könnte aber bei einem erneuten Versuch, das ethische Ernährungsbewusstsein zu fördern durchaus relevant sein.

\section{Literatur}

Block, T. (27. Oktober 2015). Ethik und Essen: Jeder Biss eine gute Tat. Südwest Presse.

Büning-Fessel, M. (2011). Ernährungskompetenz ist Lebenskompetenz. aid infodienst (Hrsg.), Du isst, wie du bist? Ernährungskompetenz ist Lebenskompetenz. Tagungsband zum 11. aid-Forum (S. 6-10). Reinheim: Lokay.

De Groot, H. (2011). Ernährungswissenschaft. Haan-Gruiten: Vollmeer. 


\section{| Ethisches Ernährungsbewusstsein}

Eberle, U. (2012). Alles jederzeit verfügbar. Lebensmittel auf Weltreise. Brockhaus Perspektiv Not für die Welt. Gütersloh: Bertelsmann.

Hirschfelder, G. \& Wittmann, B. (2015): „Was der Mensch essen darf“ - Thematische Hinführung. In G. Hirschfelder (Hrsg.), Was der Mensch essen darf. Ökonomischer Zwang, ökologisches Gewissen und globale Konflikte. Wiesbaden: Springer. https://doi.org/10.1007/978-3-658-01465-0_1

Kranert, M. (2012). Ermittlung der weggeworfenen Lebensmittelmengen und Vorschläge zur Verminderung der Wegwerfrate bei Lebensmitteln in Deutschland. Universität Stuttgart Institut für Siedlungswasserbau, Wassergüte- und Abfallwirtschaft.

Kultus und Unterricht (2008). Bildungsplan für die Berufsfachschule Lehrplanheft 2/2008. Band 1, Heft 8. Ministerium für Kultus, Jugend und Sport BadenWürttemberg (Hrsg.). Villingen-Schwenningen: Neckar-Verlag.

Kultus und Unterricht (2009). Bildungsplan für die Berufsfachschule. Lehrplan 45 6512-2220/51. 3. Band 1, Heft 1. Ministerium für Kultus, Jugend und Sport Baden-Württemberg (Hrsg.). Villingen-Schwenningen: Neckar-Verlag.

Pfeifer, V. (2009). Didaktik des Ethikunterrichts. Bausteine einer integrativen Wertevermittlung. Stuttgart: Kohlhammer.

Roth, S. J. (2014). VWL für Einsteiger. Konstanz: UVK.

Tuttle, W. (2014). Ernährung und Bewusstsein. Warum das, was wir essen, die Welt nachhaltig beeinflusst. Amerang: Crotona.

\section{Anmerkung}

1 Die Darstellung der weiteren Stunden sowie die jeweiligen Materialien können von der Autorin per E-Mail angefordert werden.

\section{Verfasserin}

Eva Steinfurth

Assessorin des Lehramtes (Lehrbefähigung in Ethik und Ernährungslehre) Master und Bachelor of Education (Höheres Lehramt an beruflichen Schulen)

Unit 405/40-44 Pakington Street

St Kilda, VIC 3182 Australia

E-Mail: e.steinfurth@yahoo.com 